Christian Grossmann Hans-Görg Roos

\title{
Numerical Treatment of Partial Differential Equations
}

Translated and revised by Martin Stynes

ei Springer 


\section{Contents}

Notation

1 Basics

$1.1 \quad$ Classification and Correctness ...........................................................

1.2 Fourier's Method, Integral Transforms .......................................... 5

1.3 Maximum Principle, Fundamental Solution ……………………... 9

1.3.1 Elliptic Boundary Value Problems ………………………... 9

1.3.2 Parabolic Equations and Initial-Boundary Value Problems ..................................................................... 15

1.3.3 Hyperbolic Initial and Initial-Boundary Value Problems 18

2 Finite Difference Methods .............................................................. 23

2.1 Basic Concepts ........................................................................ 23

2.2 Illustrative Examples .................................................................... 31

2.3 Transportation Problems and Conservation Laws ...................... 36

2.3.1 The One-Dimensional Linear Case ..................................... 37

2.3.2 Properties of Nonlinear Conservation Laws .................... 48

2.3.3 Difference Methods for Nonlinear Conservation Laws .... 53

2.4 Elliptic Boundary Value Problems ................................................... 61

2.4.1 Elliptic Boundary Value Problems ……………………...... 61

2.4.2 The Classical Approach to Finite Difference Methods 62

2.4.3 Discrete Green's Function .................................................... 74

2.4.4 Difference Stencils and Discretization in General
Domains

2.4.5 Mixed Derivatives, Fourth Order Operators .................... 82

2.4.6 Local Grid Refinements ……………………………….... 89

2.5 Finite Volume Methods as Finite Difference Schemes ................ 90

2.6 Parabolic Initial-Boundary Value Problems ................................. 103

2.6.1 Problems in One Space Dimension ................................... 104

2.6.2 Problems in Higher Space Dimensions .............................. 109

2.6.3 Semi-Discretization ............................................................. 113 
2.7 Second-Order Hyperbolic Problems …………………………........ 118

3 Weak Solutions ....................................................................................... 125

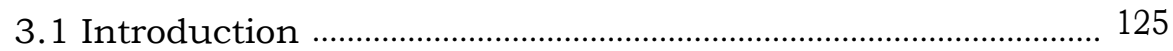

3.2 Adapted Function Spaces ……………………………………....... 128

3.3 Variational Equations and Conforming Approximation ............. 142

3.4 Weakening V-ellipticity ....................................................................... 163

3.5 Nonlinear Problems .......................................................................... 167

4 The Finite Element Method …................................................................ 173

4.1 A First Example ………………...................................................... 173

4.2 Finite-Element-Spaces ………………………………………..... 178

4.2.1 Local and Global Properties ............................................... 178

4.2.2 Examples of Finite Element Spaces in $\mathrm{R}^{2}$ and $\mathrm{R}^{3} \ldots 189$

4.3 Practical Aspects of the Finite Element Method ......................... 202

4.3.1 Structure of a Finite Element Code .................................... 202

4.3.2 Description of the Problem ................................................... 203

4.3.3 Generation of the Discrete Problem .................................... 205

4.3.4 Mesh Generation and Manipulation ................................... 210

4.4 Convergence of Conforming Methods ................................................ 217

4.4.1 Interpolation and Projection Error in Sobolev Spaces 217

4.4.2 Hilbert Space Error Estimates ............................................. 227

4.4.3 Inverse Inequalities and Pointwise Error Estimates ......... 232

4.5 Nonconforming Finite Element Methods ...................................... 238

4.5.1 Introduction .................................................................... 238

4.5.2 Ansatz Spaces with Low Smoothness ............................... 239

4.5.3 Numerical Integration ........................................................... 244

4.5.4 The Finite Volume Method Analysed from a Finite

Element Viewpoint ................................................................. 251

4.5.5 Remarks an Curved Boundaries ........................................... 254

4.6 Mixed Finite Elements ..................................................................... 258

4.6.1 Mixed Variational Equations and Saddle Points ............. 258

4.6.2 Conforming Approximation of Mixed Variational

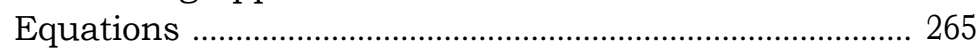

4.6.3 Weaker Regularity for the Poisson and Biharmonic

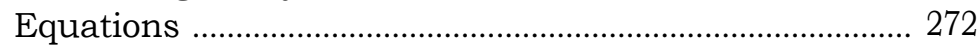

4.6.4 Penalty Methods and Modified Lagrange Functions 277

4.7 Error Estimators and Adaptive FEM ............................................. 287

4.7.1 The Residual Error Estimator ............................................... 288

4.7.2 Averaging and Goal-Oriented Estimators ......................... 292

4.8 The Discontinuous Galerkin Method ................................................ 294

4.8.1 The Primal Formulation for a Reaction-Diffusion

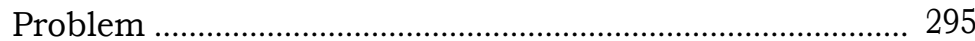

4.8.2 First-Order Hyperbolic Problems ....................................... 299

4.8.3 Error Estimates for a Convection-Diffusion Problem . . 302 
4.9 Further Aspects of the Finite Element Method ........................... 306

4.9.1 Conditioning of the Stiffness Matrix .................................. 306

4.9.2 Eigenvalue Problems ........................................................... 307

4.9.3 Superconvergence ............................................................... 310

4.9.4 p- and hp-Versions ............................................................ 314

$5 \quad$ Finite Element Methods for Unsteady Problems .................... 317

$5.1 \quad$ Parabolic Problems .......................................................................... 317

5.1.1 On the Weak Formulation ……………………………..... 317

5.1.2 Semi-Discretization by Finite Elements ............................. 321

5.1.3 Temporal Discretization by Standard Methods ............... 330

5.1.4 Temporal Discretization with Discontinuous Galerkin Methods ........................................................................... 337

5.1.5 Rothe's Method ………………………………................ 343

5.1.6 Error Control ...................................................................... 347

5.2 Second-Order Hyperbolic Problems ………………………........... 356

5.2.1 Weak Formulation of the Problem …………………......... 356

5.2.2 Semi-Discretization by Finite Elements ............................... 358

5.2.3 Temporal Discretization ........................................................ 363

5.2.4 Rothe's Method for Hyperbolic Problems ........................ 368

5.2.5 Remarks on Error Control .................................................... 372

$6 \quad$ Singularly Perturbed Boundary Value Problems ...................... 375

6.1 Two-Point Boundary Value Problems ........................................... 376

6.1.1 Analytical Behaviour of the Solution …………………....... 376

6.1.2 Discretization on Standard Meshes ……………………..... 383

6.1.3 Layer-adapted Meshes ......................................................... 394

6.2 Parabolic Problems, One-dimensional in Space ........................... 399

6.2.1 The Analytical Behaviour of the Solution ........................ 399

6.2.2 Discretization ......................................................................... 401

6.3 Convection-Diffusion Problems in Several Dimensions ............... 406

6.3.1 Analysis of Elliptic Convection-Diffusion Problems ........ 406

6.3.2 Discretization on Standard Meshes ................................... 412

6.3.3 Layer-adapted Meshes .......................................................... 427

6.3.4 Parabolic Problems, Higher-Dimensional in Space ........ 430

$7 \quad$ Variational Inequalities, Optimal Control ……………………...... 435

7.1 Analytic Properties .......................................................................... 435

7.2 Discretization of Variational Inequalities ....................................... 447

7.3 Penalty Methods ................................................................................ 457

7.3.1 Basic Concept of Penalty Methods ……………………..... 457

7.3.2 Adjustment of Penalty and Discretization Parameters .. 473

7.4 Optimal Control of PDEs ............................................................. 480

7.4.1 Analysis of an Elliptic Model Problem ............................. 480

7.4.2 Discretization by Finite Element Methods ....................... 489 
X Contents

8 Numerical Methods for Discretized Problems ................................. 499

8.1 Some Particular Properties of the Problems ................................. 499

8.2 Direct Methods ............................................................................ 502

8.2.1 Gaussian Elimination for Banded Matrices ..................... 502

8.2.2 Fast Solution of Discrete Poisson Equations, FFT ........ 504

8.3 Classical Iterative Methods ........................................................ 510

8.3.1 Basic Structure and Convergence .................................... 510

8.3.2 Jacobi and Gauss-Seidel Methods ................................... 514

8.3.3 Block Iterative Methods ...................................................... 520

8.3.4 Relaxation and Splitting Methods ................................ 524

8.4 The Conjugate Gradient Method .................................................... 530

8.4.1 The Basic Idea, Convergence Properties ........................... 530

8.4.2 Preconditioned CG Methods .............................................. 538

8.5 Multigrid Methods ........................................................................ 548

8.6 Domain Decomposition, Parallel Algorithms .......................... 560

Bibliography: Textbooks and Monographs .......................................... 571

Bibliography: Original Papers …................................................................................ 577

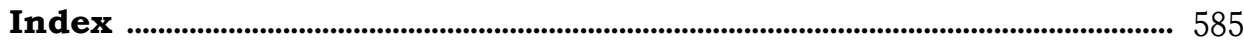

